\title{
ON THE COULOMB DISPLACEMENT ENERGY ${ }^{\dagger}$
}

\author{
HIROSHI SATO \\ Physics Department, The University of Michigan, Ann Arbor, Michigan 48109, USA \\ Received 19 January 1976 \\ (Revised 12 May 1976)
}

\begin{abstract}
The Coulomb displacement energies of the $T=\frac{1}{2}$ mirror nuclei $(A=15,17,27,29,31$, 33,39 and 41) are re-examined with the best available HF wave functions (the DME and the Skyrme II interaction), with the inclusion of all electromagnetic corrections. The results are compared with the experimental s.p. charge dependent energies extracted from the experimental data taking into account admixtures of core-excitation corrections with the help of present shell-model and co-existence model calculations. Although the so-called Nolen-Schiffer anomaly is not removed by these improvements, it is found that the remaining observed anomalies in the ground states of s.p. and s.h. systems can be resolved with the introduction of a simple, phenomenological charge symmetry breaking nucleon-nucleon force. This force can also account for the observed anomalies in the higher excited s.p. states, while those of the deeper s.h. states need further explanation.
\end{abstract}

\section{Introduction}

The Coulomb displacement energy is the binding energy difference between the ground states of mirror nuclei or between the parent and its isobaric analogue states. Under the assumption of the charge independence of the nuclear force, this is regarded as a difference between electromagnetic (e.m.) energies of these states. The Coulomb displacement energy has been studied by many authors ${ }^{1}$ ) with the employment of Coulomb interaction as the e.m. interaction. The major properties of the Coulomb displacement energy have been well understood. Moreover, fruitful information about the nuclear charge radius has been obtained through these studies.

However, with the improvement in the accuracy of the experimental data of the nuclear charge distribution and of the binding energy of the nucleus, a serious disagreement between the theoretically calculated e.m. energy difference and the experimental Coulomb displacement energy has been pointed out ${ }^{2-4}$ ). The disagreement $(4-10 \%)$ is far beyond the experimental error, and moreover it appears throughout the periodic table with the same sign. This is the so-called Nolen-Schiffer anomaly. While many attempts have been made to explain the anomaly by the inclusion of many others e.m. corrections ${ }^{5-9}$ ), such as core polarızation corrections and manybody correlations, and by the introduction of charge symmetry breaking (CSB) forces, no definite resolution of the anomaly has so far been achieved.

The difficulty with the theoretical investigation of this anomaly stems from the simultaneous uncertainty of the nature of the nucleon-nucleon $(\mathrm{N}-\mathrm{N})$ interaction

t Supported by the US National Science Foundation. 
and of the many-body structure in the nuclear wave function. Present theoretical treatments are subject to two definite shortcomings. The first stems from the single particle (s.p.) wave functions which have been used in almost all calculations of the e.m. energy. For instance, most calculations have used Woods-Saxon or harmonic oscillator wave functions. This problem may be resolved by the employment of presently available Hartree-Fock (HF) wave functions ${ }^{10}$ ). The second problem is that all calculations based on the simple shell model have compared their results with raw experimental data. However, recent detailed shell-model studies ${ }^{11,12}$ ) and earlier studies in terms of the so-called co-existence model ${ }^{13},{ }^{14}$ ) show the necessity of an appreciable admixture of core excitation in the ground-state wave functions even for closed shell plus (minus) one particle systems. The important implication of this admixture is that the experimental Coulomb displacement energy is not a pure s.p. charge dependent energy. With the resolution of these shortcomings, one may expect that any remaining observed anomaly shows some systematics as an ind!cation of its origin.

In this paper, therefore, we carefully re-examine charge dependent energies and effects of e.m. origin, employing the best available HF wave functions, and compare the results with the s.p. charge dependent energy extracted from the experimental data taking into account admixtures of core excitations with the help of present shellmodel and co-existence model calculations. Then we attempt to clarify the true anomaly in the Coulomb displacement energy and to seek the possible source of the anomaly. Throughout this work, we employ the s.p. HF wave functions obtained by the theory of the density-matrix-expansion (DME) as given by Negele and Vautherin ${ }^{15}$ ). We also compare the results with those given by the Skyrme II interaction ${ }^{16}$ ). We choose in particular those $T=\frac{1}{2}$ single particle (or hole) systems whose root mean square (rms) radii agree with those given by the DME $(A=$ $15,17,27,29,31,33,39$ and 41$)$.

In sect. 2, we determine the s.p. wave functions, which include the unbound states, by the DME, and carefully recalculate the charge dependent energies of e.m. origin and of s.p. nature. The corresponding experimental s.p. energies, with which those theoretical predictions are to be compared, are extracted from the experimental Coulomb displacement energies with the aid of presently available shell-model and co-existence model calculations in sect. 3. The net amount of the Nolen-Schiffer anomaly is then given in the same section.

In sect. 4, we show that the magnitude and the systematics of the anomaly can be explained by a phenomenological CSB N-N force, which is not inconsistent with present free $\mathrm{N}-\mathrm{N}{ }^{1} \mathrm{~S}_{0}$ scattering data.

\section{Wave functions and energies of e.m. origin \\ 2.1. DETERMINATION OF THE s.p. WAVE FUNCTION}

The neutron s.p. wave function $\varphi_{\mathrm{n}}$ is generated by the neutron HF Hamiltonian $\mathscr{H}_{\mathrm{n}}$ :

$$
\mathscr{H}_{\mathrm{n}}\left|\varphi_{\mathrm{n}}\right\rangle=\mathscr{H}_{\mathrm{n}}^{\mathrm{N}}\left|\varphi_{\mathrm{n}}\right\rangle=\varepsilon_{\mathrm{n}}\left|\varphi_{\mathrm{n}}\right\rangle,
$$


where $\mathscr{H}_{\mathrm{n}}^{\mathrm{N}}$ is the nuclear part of the neutron HF Hamiltonian. The proton s.p. wave function $\left|\varphi_{\mathrm{p}}\right\rangle$ is also necessary in the calculations of the second order Coulomb perturbation and of the core polarization effect. This is generated by the proton HF Hamiltonian $\mathscr{H}_{\mathrm{p}}$ :

$$
\mathscr{H}_{\mathrm{p}}\left|\varphi_{\mathrm{p}}\right\rangle=\left(\mathscr{H}_{\mathrm{p}}^{\mathrm{N}}+U_{\mathrm{c}}\right)\left|\varphi_{\mathrm{p}}\right\rangle=\varepsilon_{\mathrm{p}}\left|\varphi_{\mathrm{p}}\right\rangle,
$$

where $\mathscr{H}_{\mathrm{p}}^{\mathrm{N}}$ and $U_{\mathrm{c}}$ are respectively the nuclear part of the proton HF Hamiltonian and its Coulomb part. The Hamiltonians $\mathscr{H}_{\mathrm{n}}$ and $\mathscr{H}_{\mathrm{p}}$ are generated by the DME (or the Skyrme II interaction) code given by Negele and Vautherin ${ }^{17}$ ).

There exists a small difference between the $\mathscr{H}_{\mathrm{n}}^{\mathrm{N}}$ and $\mathscr{H}_{\mathrm{p}}^{\mathrm{N}}$. The effect of this difference is taken into consideration in terms of the core polarization correction ${ }^{\mathbf{1 8}}$ ).

TABLE 1

The s.p. energies $\varepsilon_{\mathrm{n}}, \varepsilon_{\mathrm{p}}$ and rms charge radii obtained by the DME and the Skyrme II interaction, and the experimental data for the ${ }^{16} \mathrm{O}$ and ${ }^{40} \mathrm{Ca}$ core system

\begin{tabular}{|c|c|c|c|c|c|c|c|}
\hline \multicolumn{5}{|c|}{ Neutrons } & \multicolumn{3}{|c|}{ Protons } \\
\hline & state & DME & SKII & $\left.\exp { }^{4}\right)$ & DME & SKII & $\left.\exp ^{4}\right)$ \\
\hline \multirow[t]{7}{*}{${ }^{16} \mathrm{O}$} & $I s_{\frac{1}{2}}$ & -38.63 & -41.40 & & -34.98 & -37.71 & $-40 \pm 8$ \\
\hline & $1 p_{\frac{3}{4}}$ & -19.99 & -21.09 & -21.8 & -16.60 & -17.64 & -18.4 \\
\hline & $1 p_{\frac{1}{2}}$ & -14.19 & -15.92 & -15.7 & -10.94 & -12.56 & -12.1 \\
\hline & $1 d_{\frac{8}{2}}$ & -4.00 & -4.00 & -4.17 & -1.01 & -0.90 & -0.60 \\
\hline & $2 s_{\frac{1}{2}}$ & -2.58 & -1.57 & -3.27 & 0.03 & 0.87 & -0.10 \\
\hline & $1 d_{\frac{3}{2}}$ & 1.47 & 1.43 & & 4.20 & 4.31 & \\
\hline & $r_{\mathrm{rms}}$ & & & & 2.768 & 2.707 & 2.73 \\
\hline \multirow[t]{11}{*}{${ }^{40} \mathrm{Ca}$} & $1 s_{\frac{1}{2}}$ & -52.42 & -55.07 & & -44.73 & -47.38 & $-50 \pm 11$ \\
\hline & $1 p_{\frac{3}{2}}$ & -37.33 & -38.84 & & -29.93 & -31.44 & $-34 \pm 6$ \\
\hline & $1 p_{\frac{1}{2}}$ & -33.39 & -35.74 & & -26.08 & -28.40 & \\
\hline & $1 d_{\frac{3}{2}}^{2}$ & -22.18 & -22.75 & & -15.13 & -15.68 & \\
\hline & $2 s_{\frac{1}{2}}$ & -17.16 & -16.67 & -18.1 & -10.22 & -9.62 & -10.9 \\
\hline & $1 d_{\frac{3}{2}}$ & -15.51 & -17.13 & -15.6 & -8.67 & -10.17 & -8.3 \\
\hline & $1 \mathrm{f}_{\frac{7}{2}}$ & -7.73 & -7.73 & -8.36 & -1.17 & -1.07 & -1.4 \\
\hline & $2 p_{\frac{3}{2}}$ & -3.95 & -2.69 & -6.2 & 1.95 & 3.11 & \\
\hline & $2 \mathrm{p}_{\frac{1}{2}}$ & -2.13 & -1.30 & & 3.35 & 4.39 & \\
\hline & $1 f_{\frac{3}{2}}$ & -0.07 & -0.79 & & 5.81 & 5.39 & \\
\hline & $r_{\text {rens }}$ & & & & 3.481 & 3.464 & 3.49 \\
\hline
\end{tabular}

*) Ref. ${ }^{16}$ ). All energies are in units of $\mathrm{MeV}$, and rms radii are in unis of $\mathrm{fm}$.

Table 1 shows the s.p. energies $\varepsilon_{\mathrm{n}}, \varepsilon_{\mathrm{p}}$ and rms charge radii obtained with the DME and the Skyrme II interaction for the ${ }^{16} \mathrm{O}$ and ${ }^{40} \mathrm{Ca}$ core systems. The experimental numbers are also shown in the table.

In the case of the unbound s.p. states, such as the proton $2 s_{\frac{1}{2}}, 1 d_{\frac{2}{2}}$ and neutron $1 d_{\frac{3}{2}}$ states for the ${ }^{16} \mathrm{O}$ core, and the proton $2 \mathrm{p}_{\frac{3}{2}}, 2 \mathrm{p}_{\frac{1}{4}}$ and $1 \mathrm{f}_{\frac{2}{2}}$ states for the ${ }^{40} \mathrm{Ca}$ core, we 
connect the radial wave function of the irregular Coulomb wave functions $G_{l}(k r)$ [ref. $\left.{ }^{19}\right)$ ] in the region far beyond the nuclear field:

$$
R_{l}(r) \sim G_{l}(k r),
$$

so that the asymptotic form corresponds to a $\frac{1}{2} \pi$ phase shift at the resonance energy. The calculational method of Buck and Hodgson ${ }^{20}$ ) is used to make the smooth connection. While there exist several methods ${ }^{21}$ ) for the normalization of the unbound state, we employ the following normalization method. The normalized s.p. wave function $|\varphi\rangle^{\text {norm }}$ is related to the unnormalized wave functions $|\varphi\rangle$ in the following manner:

$$
\begin{gathered}
|\varphi\rangle^{\text {norm }}=|\varphi\rangle / \sqrt{\sum\left|C_{n}\right|^{2}}, \\
|\varphi\rangle=\sum_{n=0}^{N_{\max }} C_{n}\left|\varphi_{n}^{\mathrm{HO}}\right\rangle,
\end{gathered}
$$

where $C_{n}$ is the expansion coefficient and $\left|\varphi_{n}^{\text {HO }}\right\rangle$ is the normalized harmonic oscillator wave function having the same angular momentum. The harmonic oscillator constant is adjusted to give the same rms charge radius. The dependence of the normalization constant on the choice of $N_{\max }$ is negligible for values of $N_{\max } \geqq 10$, and $N_{\max }$ is chosen to have the value 10 . On the other hand, this normalization method is effectively the same as one in which the unbound state wave function is limited to a specific range. For instance, the value 10 of $N_{\max }$ corresponds to normalization within a range of $14 \mathrm{fm}$ for the ${ }^{40} \mathrm{Ca}$ core system and of $12.5 \mathrm{fm}$ for the ${ }^{16} \mathrm{O}$ core. Therefore, the integration in the calculation of the matrix element is undertaken within the same range.

\subsection{ENERGIES OF e.m. ORIGIN}

Employing the s.p. wave function obtained with the DME, s.p. charge dependent energies of e.m. origin and other charge dependent corrections of s.p. nature have been calculated for the s.p. and s.h. systems with the use of the multipole expansion of the force ${ }^{22,23}$ ). The results are summarized in table 2, and compared with the experimental Coulomb displacement energies of the lowest states of the assigned spin and parity. The calculated charge dependent energies are the Coulomb energy $\varepsilon_{\mathrm{C}}$, the proton finite size correction $\varepsilon_{\mathrm{f} . \mathrm{s.p} \text {. }}$ [ref. ${ }^{24}$ )], the e.m. spin-orbit interaction $\varepsilon_{\mathrm{s.o} .}^{\text {e.m. }}$, the vacuum polarization correction $\varepsilon_{\mathrm{v} \text {.p. }}$, the short range correlational correction $\varepsilon_{\text {s.r.c. }}$, the core polarization correction $\varepsilon_{\text {c.p. }}$, the kinetic energy correction $\varepsilon_{\text {k.e.c. }}$ due to the mass difference between the bare neutron and the bare proton, and the Coulomb perturbation energy $\varepsilon_{\mathrm{C}}^{\text {pex }}$. Here the vacuum polarization correction is estimated with the method given by Auerbach et al. ${ }^{7}$ ). The short range correlational correction is obtained with the use of matrix elements estimated by Bertsch and Shlomo ${ }^{25}$ ). The core polarization correction is estimated with the method given by Giai et al. ${ }^{18}$ ). The Coulomb perturbation energy is calculated with the method given by Arima and Yoshida ${ }^{26}$ ). 
TABLE 2

The s.p. charge dependent energies (keV) calculated with the DME and the experımental Coulomb displacement energies of lowest states of the assigned spin and parity

\begin{tabular}{|c|c|c|c|c|c|c|c|c|c|c|c|c|}
\hline $\boldsymbol{A}$ & State & $\varepsilon_{\mathrm{C}}$ & $\varepsilon_{\text {f.s.p. }}$ & $\begin{array}{l}\varepsilon_{\text {s.o. }}^{\text {e.m. }} \\
\text { nats }\end{array}$ & $\varepsilon_{\mathrm{v}, \mathrm{p}}$ & $\varepsilon_{\text {s.r. }}$ & $\varepsilon_{\text {c.p. }}$ & $\varepsilon_{k, \text { e.c. }}$ & $\varepsilon_{\mathbf{C}}^{\text {per }}$ & Total & $\left.\operatorname{Exp}^{2}\right)$ & Diff. \\
\hline \multirow[t]{2}{*}{15} & $1 \mathrm{p}_{\frac{3}{2}}-1$ & 3275 & -80 & -35 & 20 & 125 & -100 & 25 & -15 & 3215 & 3395 & 180 \\
\hline & $1 \mathrm{p}_{\frac{1}{2}}-1$ & 3200 & -75 & 70 & 20 & 105 & -140 & 20 & -20 & 3180 & 3542 & 360 \\
\hline \multirow[t]{3}{*}{17} & $1 \mathrm{~d}_{\frac{8}{2}}^{2}$ & 3195 & -50 & -60 & 20 & 85 & 15 & 30 & -35 & 3200 & 3542 & 340 \\
\hline & $2 s_{\frac{1}{2}}^{2}$ & 2910 & -40 & 0 & 15 & 95 & 25 & 20 & -120 & 2905 & 3166 & 260 \\
\hline & $1 d_{\frac{3}{2}}^{\frac{2}{2}}$ & 2495 & -25 & 50 & 15 & 55 & 285 & 15 & -15 & 2875 & 3562 & 685 \\
\hline 27 & $1 \mathrm{~d}_{\frac{5}{2}}^{\frac{2}{2}}-1$ & 5165 & -85 & -95 & 30 & 125 & 0 & 30 & -40 & 5130 & 5592 & 460 \\
\hline 29 & $2 s_{\frac{1}{2}}^{2}$ & 5475 & -80 & -20 & 35 & 130 & -90 & 25 & -60 & 5415 & 5762 & 310 \\
\hline 31 & $2 s_{\frac{1}{2}}^{2}-1$ & 5795 & -90 & -20 & 35 & 145 & -105 & 25 & -75 & 5710 & 6224 & 515 \\
\hline 33 & $1 d_{\frac{3}{2}}^{2}$ & 6015 & -80 & 100 & 35 & 120 & -155 & 25 & -70 & 5990 & 6365 & 375 \\
\hline \multirow[t]{2}{*}{39} & $2 s_{\frac{1}{2}}^{2}-1$ & 7085 & -100 & 0 & 45 & 160 & -195 & 25 & -80 & 6940 & 7253 & 315 \\
\hline & $1 d_{\frac{3}{2}}^{2}-1$ & 6955 & -95 & 115 & 40 & 125 & -205 & 25 & -60 & 6895 & 7304 & 410 \\
\hline \multirow[t]{4}{*}{41} & $1 \mathrm{f}_{\frac{7}{2}}^{2}$ & 6775 & -70 & -105 & 40 & 120 & 70 & 35 & -75 & 6790 & 7278 & 490 \\
\hline & $2 \mathrm{p}_{\frac{3}{2}}^{2}$ & 6465 & -65 & -30 & 40 & 125 & 180 & 25 & -410 & 6330 & 7051 & 750 \\
\hline & $2 \mathrm{p}_{\frac{1}{2}}^{2}$ & 6075 & -55 & 55 & 35 & 105 & 290 & 20 & -545 & 5980 & $\left.6803^{b}\right)$ & 825 \\
\hline & $1 \mathrm{f}_{\frac{1}{3}}^{2}$ & 6235 & -55 & 120 & 35 & 75 & 180 & 25 & -260 & 6355 & & \\
\hline
\end{tabular}

a) $A=15$; ref. ${ }^{27}$ ). $A=17$; ref. ${ }^{28}$ ). $A=27-41$; ref. ${ }^{29}$ ).

b) Refs. ${ }^{30.31}$ ).

To examine the model dependence of the results, we compare the values obtained with the DME with those given by the Skyrme II interaction in table 3. In this table, we show only the energies $\varepsilon_{\mathrm{C}}, \varepsilon_{\text {c.p. }}$. $\varepsilon_{\mathrm{C}}^{\text {per }}$ and $\varepsilon_{\text {total }}$, because the other corrections $\varepsilon_{\text {f.s.p. }}$, $\varepsilon_{s .0 .}^{\text {e.m. }}, \varepsilon_{\mathrm{v} . p .}, \varepsilon_{\mathrm{s}}$ r.c. and $\varepsilon_{\text {k.e.c. }}$ do not change significantly. In general, the Skyrme II interaction gives larger Coulomb energies $\varepsilon_{\mathrm{C}}$ for the states of zero node. This stems from

TABLE 3

Comparison of the s.p. energies (keV) calculated with the DME with those obtained with the Skyrme II interaction

\begin{tabular}{|c|c|c|c|c|c|c|c|c|c|}
\hline \multicolumn{6}{|c|}{ DME } & \multicolumn{4}{|c|}{ Skyrme II } \\
\hline$A$ & state & $\varepsilon_{\mathbf{C}}$ & $\varepsilon_{\text {e.p. }}$ & $\varepsilon_{\mathbf{C}}^{\text {per }}$ & $\varepsilon_{\text {total }}$ & $\varepsilon_{c}$ & $\varepsilon_{\text {c.p. }}$ & $\varepsilon_{\mathbf{C}}^{\text {per }}$ & $\varepsilon_{\text {total }}$ \\
\hline 15 & $\begin{array}{l}1 p_{\frac{2}{2}}-1 \\
1 p_{\frac{1}{2}}^{-1}\end{array}$ & $\begin{array}{l}3275 \\
3200\end{array}$ & $\begin{array}{l}-100 \\
-140\end{array}$ & $\begin{array}{l}-15 \\
-20\end{array}$ & $\begin{array}{l}3215 \\
3180\end{array}$ & $\begin{array}{l}3320 \\
3280\end{array}$ & $\begin{array}{l}-95 \\
-130\end{array}$ & $\begin{array}{l}-10 \\
-15\end{array}$ & $\begin{array}{l}3270 \\
3270\end{array}$ \\
\hline 17 & $\begin{array}{l}1 d_{\frac{5}{2}} \\
2 s_{\frac{1}{2}} \\
1 d_{\frac{3}{2}}\end{array}$ & $\begin{array}{l}3195 \\
2910 \\
2495\end{array}$ & $\begin{array}{r}15 \\
25 \\
285\end{array}$ & $\begin{array}{l}-35 \\
-120 \\
-15\end{array}$ & $\begin{array}{l}3200 \\
2905 \\
2875\end{array}$ & $\begin{array}{l}3260 \\
2785 \\
2610\end{array}$ & $\begin{array}{r}55 \\
150 \\
315\end{array}$ & $\begin{array}{l}-30 \\
-265 \\
-\quad 15\end{array}$ & $\begin{array}{l}3305 \\
2760 \\
3030\end{array}$ \\
\hline 39 & $\begin{array}{l}2 s_{\frac{2}{2}}-1 \\
1 d_{\frac{3}{2}}-1\end{array}$ & $\begin{array}{l}7085 \\
6955\end{array}$ & $\begin{array}{l}-195 \\
-205\end{array}$ & $\begin{array}{l}-80 \\
-65\end{array}$ & $\begin{array}{l}6940 \\
6895\end{array}$ & $\begin{array}{l}7090 \\
6990\end{array}$ & $\begin{array}{l}-125 \\
-155\end{array}$ & $\begin{array}{l}-60 \\
-45\end{array}$ & $\begin{array}{l}7040 \\
7000\end{array}$ \\
\hline 41 & $\begin{array}{l}1 f_{\frac{7}{2}} \\
2 p_{\frac{3}{3}} \\
1 p_{\frac{1}{3}} \\
1 f_{\frac{5}{2}}\end{array}$ & $\begin{array}{l}6775 \\
6465 \\
6075 \\
6235\end{array}$ & $\begin{array}{r}70 \\
180 \\
290 \\
180\end{array}$ & $\begin{array}{l}-75 \\
-410 \\
-545 \\
-260\end{array}$ & $\begin{array}{l}6790 \\
6330 \\
5980 \\
6355\end{array}$ & $\begin{array}{l}6780 \\
6270 \\
5830 \\
6405\end{array}$ & $\begin{array}{l}140 \\
485 \\
385 \\
275\end{array}$ & $\begin{array}{l}-60 \\
-595 \\
-320 \\
-245\end{array}$ & $\begin{array}{l}6875 \\
6260 \\
6055 \\
6645\end{array}$ \\
\hline
\end{tabular}


large rms charge radii obtained with the Skyrme II interaction. The DME gives larger Coulomb energies $\varepsilon_{\mathrm{C}}$ for the states of higher node. This is related to the model dependence of the s.p. energies shown in table 1 . The $\varepsilon_{\text {c.p. }}$ is dependent upon the details of the model rather than the binding energies obtained. On the other hand, the $\varepsilon_{\mathrm{C}}^{\text {per }}$ is only dependent upon the binding energies $\varepsilon_{\mathrm{n}}$ and $\varepsilon_{\mathrm{p}}$.

\section{Core-excitation correction}

Thus far we have examined the corrections of s.p. nature. On the other hand, recent detailed shell-model studies and earlier studies in terms of the so-called coexistence model indicate significant admixtures of core excitation in the ground-state wave functions even for closed shell plus (minus) one particle systems. The experimentally observed Coulomb displacement energies for the lowest states are therefore not the pure s.p. (s.h.) charge dependent energies. The effect of the core excitation could be handled as a correction to the calculated s.p. (s.h.) charge dependent energy. However, we prefer instead to consider the presence of the core excitation as implying a change in the interpretation of the experimental data and use our knowledge about the core excitations to extract an experimental s.p. charge dependent energy from the experimentally observed Coulomb displacement energies.

\subsection{GENERAL TREATMENT}

In the s.p. systems the $T=\frac{1}{2}$ states of a particular spin and parity have wave functions which can be expressed in terms of the s.p. wave function and particle-hole excitations as follows:

$$
\left|\alpha^{2}\right\rangle=\sqrt{1-\sum_{s}\left|C_{s}^{i}\right|^{2}}|\alpha: 1 \mathrm{p}\rangle+\sum_{s} C_{s}^{i}\left|\alpha:\left(n_{s} \mathrm{p}-\left(n_{s}-1\right) \mathrm{h}\right)\right\rangle,
$$

where the subscript $s$ specifies the core excitation and the superscript $i$ is the identification number of the state startıng with $i=1$ for the lowest state. In general the state with $i=1$ has a predominant s.p. component, whereas the core excitations are the major components in states with $i=2,3, \ldots$. For the truncated $\mathrm{p}-\mathrm{h}$ space, with $N$ p-h excitations, the amplitudes $C_{s}^{i}$ satisfy the relationship,

$$
\sum_{i}^{N+1}\left|C_{s}^{i}\right|^{2}=1
$$

where $N=\sum_{s}^{s_{N}} 1$, and where the summation of $s$ is undertaken over all the possible core excitations up to $s_{N}$.

The charge dependent energy $\mathscr{E}^{i}(\alpha)$ of the state $\left|\alpha^{i}\right\rangle$ is defined by the binding energy difference,

$$
\mathscr{E}^{i}(\alpha)=-\left[\text { B.E. }\left(\alpha^{i}\right)\right]_{T_{z}=-\frac{1}{2}}+\left[\text { B.E. }\left(\alpha^{i}\right)\right]_{T_{z}=\frac{1}{2}} .
$$

Using the wave function (3.1), $\mathscr{E}^{i}(\alpha)$ is given by

$$
\mathscr{E}^{\prime}(\alpha)=\varepsilon(\alpha)+\sum_{s}\left|C_{s}^{i}\right|^{2} \Delta_{s}^{\mathrm{CE}}(\alpha)
$$


where $\varepsilon(\alpha)$ is the total s.p. charge dependent energy of the state $\alpha$ and the $\Delta_{s}^{\mathrm{CE}}(\alpha)$ is the calculated Coulomb energy correction for the $s$-type core-excitation relative to $\varepsilon(\alpha)$, [see eq. (3.8) below]. Here we neglect the cross terms between two different core-excited states. Thus, the s.p. charge dependent energy $\varepsilon(\alpha)$ can be obtained from eq. (3.4) in those cases where theoretical model wave functions are available to give an estimate of the $C_{s}^{i}$. On the other hand, using the relationship (3.2), we can determine the s.p. charge dependent energy $\varepsilon(\alpha)$ from the experimental $\mathscr{E}^{i}(\alpha)$ and the calculated $\Delta_{s}^{\mathrm{CE}}$ by

$$
\varepsilon(\alpha)=\frac{1}{N+1}\left(\sum_{i=1}^{N+1} \mathscr{E}^{\infty i}(\alpha)-\sum_{s}^{s_{N}} \Delta_{s}^{\mathrm{CE}}(\alpha)\right)
$$

provided the assignment of the experimental levels $i=1, \ldots, N+1$ is consistent with the core excitations implied by the states $s$ in eq. (3.1). This method has the additional advantage that cross terms between two different core-excited states (which were neglected above) are cancelled out.

In this work, we employ the above two methods to determine the s.p. charge dependent energy $\varepsilon(\alpha)$. In the first method, based on eq. (3.4), the $\varepsilon(\alpha)$ are determined from the single experimental number, $\mathscr{E}^{t=1}(\alpha)$, and knowledge of the $C_{s}^{i=1}$ from theoretical methods. In the second method, based on eq. (3.5), the $\varepsilon(\alpha)$ are extracted from the full set of experimental numbers, $\mathscr{E}^{i}(\alpha)(i=1,2$ or $1,2,3, \ldots)$, with the help of the calculated quantities, $\Delta_{s}^{\mathrm{CE}}(\alpha)$, which are less model dependent than the $C_{s^{*}}^{i}$

In addition, we have the following methods for the verification of the reliability of these $\varepsilon(\alpha)$. If the spectroscopic factor $S_{\alpha}^{l}$ is known experimentally, we can determine rough upper and lower limits for the s.p. charge dependent energy $\varepsilon(\alpha)$ :

$$
\mathscr{E}^{i}(\alpha)-\left(1-S_{\alpha}^{i}\right) \Delta_{s_{\max }}^{\mathrm{CE}}(\alpha) \leqq \varepsilon(\alpha) \leqq \mathscr{E}^{i}(\alpha)-\left(1-S_{\alpha}^{i}\right) \Delta_{s_{\min }}^{\mathrm{CE}}(\alpha),
$$

where the $\Delta_{s_{\max }}^{\mathrm{CE}}(\alpha)$ is the largest core-excitation correction and the $\Delta_{s_{\min }}^{\mathrm{CE}}(\alpha)$ is the smallest one. Moreover, combining eqs. (3.4) and (3.5), we have a consistency relationship

$$
\sum_{s}^{s_{N}}\left|C_{s}^{i}\right|^{2} \Delta_{s}^{\mathrm{CE}}(\alpha)=\mathscr{E}(\alpha)-\frac{1}{N+1}\left(\sum_{j=1}^{N+1} \mathscr{E}^{j}(\alpha)-\sum_{s}^{s_{N}} \Delta_{s}^{\mathrm{CE}}(\alpha)\right),
$$

which, when satisfied, can serve as a strong criterion for the validation of the model wave functions.

\subsection{CORRECTIONS FOR CORE EXCITATIONS $\left(\Delta_{s}^{\mathrm{CE}}\right)$}

We assume that the most important core-excited states in the $T=\frac{1}{2}$ s.p. systems are the $3 \mathrm{p}-2 \mathrm{~h}$ states with the particle isospin $T_{\mathrm{p}}=\frac{1}{2}$. In the case of the ${ }^{16} \mathrm{O}$ and ${ }^{40} \mathrm{Ca}$ core systems, we also take into account a $5 \mathrm{p}-4 \mathrm{~h}$ state with isospins $\left(T_{\mathrm{p}}, T_{\mathrm{b}}, T\right)=\left(\frac{1}{2}\right.$, $0, \frac{1}{2}$ ). Although the corrections due to $2 \mathrm{p}-1 \mathrm{~h}$ states of $2 h \omega$ excitation are neglected, some of the effects of these $\mathrm{p}-\mathrm{h}$ excitations are contained in our s.p. wave functions 
since they are folded into the HF wave function ${ }^{32}$ ). We also assume that the particle states $p$ and the hole states $h$ in core-excitation are constructed from the spherical shell-model states nearest to the Fermi level, and the coupling of their angular momenta is neglected. For the $T=\frac{1}{2} \mathrm{~s}$.h. systems, we simply interchange the roles of the particle and the hole.

The correction $\Delta_{s}^{\mathrm{CE}}(\alpha)$ for the $s$-type core excitation relative to the single particle state $\alpha$ is defined by

$$
\Delta_{s}^{\mathrm{CE}}(\alpha) \equiv E^{\mathrm{CDE}}(\alpha: s)-E^{\mathrm{CDE}}(\alpha: 1 \mathrm{p}),
$$

where $E^{\mathrm{CDE}}(\alpha: s)$ is the Coulomb displacement energy of the s-type core-excited state $\alpha$ and $E^{\mathrm{CDE}}(\alpha: 1 \mathrm{p})$ is that of the s.p. state $\alpha$. The correction $\Delta^{\mathrm{CE}}(\alpha)$ has a different form for each type of core excitation.

(i) The correction to the Coulomb displacement energy for the $3 \mathrm{p}-2 \mathrm{~h}$ state $\left(s_{1}\right)$ with isospins $\left(\frac{1}{2}, 1, \frac{1}{2}\right)$ relative to the $1 \mathrm{p}-0 \mathrm{~h}$ state $\alpha$ is

$\Delta_{s_{1}}^{\mathrm{CE}}(\alpha)=\varepsilon(\mathrm{p})-\varepsilon(\alpha)-\frac{2}{3}\left(2 \varepsilon(\mathrm{p})-2 \varepsilon(\mathrm{h})-4 V_{\mathrm{phph}}+V_{\mathrm{pppp}}+V_{\mathrm{hhhb}}\right)+\frac{1}{3}\left(V_{\mathrm{pppp}}-V_{\mathrm{phph}}\right)$.

The correction for the $2 \mathrm{p}-3 \mathrm{~h}$ state with isospins $\left(1, \frac{1}{2}, \frac{1}{2}\right)$ relative to the $0 \mathrm{p}-1 \mathrm{~h}$ state $q$ is

$\Delta_{s_{1}}^{\mathrm{CE}}(q)=\varepsilon(\mathrm{h})-\varepsilon(q)+\frac{2}{3}\left(2 \varepsilon(\mathrm{p})-2 \varepsilon(\mathrm{h})-4 V_{\mathrm{phph}}+V_{\mathrm{pppp}}+V_{\mathrm{hhhh}}\right)+\frac{1}{3}\left(V_{\mathrm{phph}}-V_{\mathrm{bhhh}}\right)$

(ii) The correction for the $3 \mathrm{p}-2 \mathrm{~h}$ state $\left(s_{2}\right)$ with isospins $\left(\frac{1}{2}, 0, \frac{1}{2}\right)$ relative to the $1 \mathrm{p}$ state $\alpha$ is

$$
\Delta_{s_{2}}^{\mathrm{CE}}(\alpha)=\varepsilon(p)-\varepsilon(\alpha)+\left(V_{\mathrm{pppp}}-V_{\mathrm{phph}}\right) .
$$

The correction for the $2 \mathrm{p}-3 \mathrm{~h}$ state with isospins $\left(0, \frac{1}{2}, \frac{1}{2}\right)$ relative to the $1 \mathrm{~h}$ state $q$ is

$$
\Delta_{s_{2}}^{\mathrm{CE}}(q)=\varepsilon(\mathrm{h})-\varepsilon(q)+\left(V_{\text {phph }}-V_{\text {hhhh }}\right) \text {. }
$$

(iii) The correction for the $5 \mathrm{p}-4 \mathrm{~h}$ state $\left(s_{3}\right)$ with isospins $\left(\frac{1}{2}, 0, \frac{1}{2}\right)$ relative to the $1 \mathrm{p}$ state $\alpha$ is

$$
\Delta_{s_{3}}^{\mathrm{CE}}(\alpha)=\varepsilon(\mathrm{p})-\varepsilon(\alpha)+2\left(V_{\mathrm{pppp}}-V_{\mathrm{phph}}\right)
$$

The correction for the $4 p-5 h$ state with isospins $\left(0, \frac{1}{2}, \frac{1}{2}\right)$ relative to the $1 \mathrm{~h}$ state $q$ is

$$
\Delta_{s_{3}}^{\mathrm{CE}}(q)=\varepsilon(\mathrm{h})-\varepsilon(q)+2\left(V_{\mathrm{phph}}-V_{\mathrm{hhhh}}\right) \text {. }
$$

The calculated corrections are shown in table 4. Here we employ the total s.p. charge dependent energies obtained in the previous section for the $\varepsilon$-contributions to the correction. For the two-body part, $V$, we take simply the angular momentumaveraged Coulomb matrix elements.

From eqs. (3.5) and (3.9) to (3.11) and the essential $\alpha$-independence of the $V$ matrix elements, it can be seen that the difference between two s.p. energies must be related to the experimental Coulomb displacement energies by

$$
\varepsilon(\alpha)-\varepsilon(\beta)=\sum_{i=1}^{N+1}\left(\mathscr{E}^{i}(\alpha)-\mathscr{E}^{i}(\beta)\right)
$$


TABLE 4

Coulomb displacement energies for the core excitations relative to the lp-0h (0p-lh) state

\begin{tabular}{llrrr}
\hline$A$ & State & \multicolumn{1}{c}{$\Delta_{s_{1}}$ CE } & \multicolumn{1}{c}{$\Delta_{\mathbf{s}_{2}}$ CE } & \multicolumn{1}{c}{$\Delta_{\mathbf{s}_{3}}$ CE } \\
\hline 15 & $1 \mathrm{p}_{\frac{3}{2}}-1$ & -540 & -100 & 0 \\
& $1 \mathrm{p}_{\frac{1}{2}}-1$ & -505 & -65 & 0 \\
17 & $1 \mathrm{~d}_{\frac{3}{2}}$ & 475 & -35 & -65 \\
& $2 \mathrm{~s}_{\frac{1}{2}}$ & 770 & 260 & 230 \\
& $1 \mathrm{~d}_{\frac{3}{2}}$ & 800 & 290 & 260 \\
27 & $1 \mathrm{~d}_{\frac{3}{2}}-1$ & -85 & -15 & -30 \\
29 & $2 \mathrm{~s}_{\frac{1}{2}}$ & 90 & 25 & 0 \\
31 & $2 \mathrm{~s}_{\frac{1}{2}}-1$ & -80 & -35 & 0 \\
33 & $1 \mathrm{~d}_{\frac{3}{2}}$ & 70 & 5 & 5 \\
39 & $2 \mathrm{~s}_{\frac{1}{2}}-1$ & -640 & -60 & -75 \\
& $1 \mathrm{~d}_{\frac{3}{2}}-1$ & -595 & -15 & -30 \\
41 & $1 \mathrm{f}_{\frac{7}{2}}$ & 585 & -20 & -40 \\
& $2 \mathrm{p}_{\frac{3}{2}}$ & 1045 & 440 & 420 \\
& $2 \mathrm{p}_{\frac{1}{2}}$ & 1395 & 790 & 770 \\
& $1 \mathrm{f}_{\frac{1}{2}}$ & 1020 & 415 & 395 \\
\hline
\end{tabular}

The $\Delta_{s} \mathrm{cE}$ are calculated by the DME and are in units of keV.

For $T=\frac{1}{2}$, s.p. systems:

(1) $s_{1}=$ the $3 \mathrm{p}-2 \mathrm{~h}$ state with isosopins $\left(\frac{1}{2}, 1, \frac{1}{2}\right)$,

(i1) $s_{2}=$ the $3 \mathrm{p}-2 \mathrm{~h}$ state with isospins $\left(\frac{1}{2}, 0 \frac{1}{2}\right)$,

(nii) $s_{3}=$ the $5 \mathrm{p}-4 \mathrm{~h}$ state with isospins $\left(\frac{1}{2}, 0, \frac{1}{2}\right)$.

For $T=\frac{1}{2}$ s.h. systems, the roles of the particle and the hole are interchanged.

where both states $\alpha$ and $\beta$ must be either particle states or hole states. Although the derivation of the relationship (3.12) depends upon each correction term, its final form does not include the correction terms. Therefore, this relationship can serve as another strong criterion for the validation of the model.

\subsection{DETERMINATION OF THE s.p. ENERGY}

Using the calculated corrections for core excitations of table 4, the s.p. charge dependent energies have been calculated by the two methods outlined in subsect. 3.1. The results are summarized in table 5 . The first column of this table shows the experimental excitation energy $E_{\mathrm{ex}}^{i}(\alpha)$ of the state $\left|\alpha^{i}\right\rangle$ (in the nucleus with $T_{z}=-\frac{1}{2}$ ). The second column gives the experimental Coulomb displacement energy $\mathscr{E}^{\sigma_{1}}(\alpha)$ of the state $\left|\alpha^{i}\right\rangle$. The third column, $\varepsilon^{\text {av }}(\alpha)$, shows the s.p. charge dependent energy which is determined from the average Coulomb displacement energy of the first $N+1$ levels by eq. (3.5) using the DME. The number in parenthesis is obtained using the Skyrme II interaction. The core excitations in this averaging are denoted by the $s_{\mathfrak{n}}$ in the next column. Here $s_{1}$ is the $3 \mathrm{p}-2 \mathrm{~h}$ state with isospins $\left(T_{\mathrm{p}}, T_{\mathrm{h}}, \mathrm{T}\right)=\left(\frac{1}{2}, 1, \frac{1}{2}\right), s_{2}$ is the $3 \mathrm{p}-2 \mathrm{~h}$ state with $\left(\frac{1}{2}, 0, \frac{1}{2}\right)$ and $s_{3}$ is the $5 \mathrm{p}-4 \mathrm{~h}$ state with $\left(\frac{1}{2}, 0, \frac{1}{2}\right)$. For the s.h. system, we simply interchange the roles of the particle and the hole. The fifth column, $\varepsilon^{\text {theor }}(\alpha)$, shows the s.p. charge dependent energy obtained by eq. (3.4) using the best available model wave function and the core-excitation correction generated by the DME. The 
TABLE 5

The s.p. charge dependent energies extracted from the experımental Coulomb displacement energies

\begin{tabular}{|c|c|c|c|c|c|c|c|c|c|}
\hline \multirow[t]{2}{*}{$\boldsymbol{A}$} & \multirow[t]{2}{*}{ State } & \multirow[t]{2}{*}{$E_{\text {ex }}{ }^{(a)}$} & \multirow[t]{2}{*}{$\left.\mathscr{E}^{i}(\alpha)^{a}\right)$} & \multicolumn{3}{|c|}{$\varepsilon^{\mathrm{av}}(\alpha)$} & \multicolumn{2}{|c|}{$\varepsilon^{\text {theor }}(\alpha)$} & \multirow[t]{2}{*}{$\varepsilon^{\mathrm{opt}}(\alpha)$} \\
\hline & & & & DME & (SkII) & type & $\overline{\mathrm{DME}}$ & $\overline{\text { (SkII) }}$ & \\
\hline \multirow[t]{6}{*}{15} & $\frac{3}{2}^{-}$ & 6.32 & 3395 & & & & 3465 & $(3460)$ & 3460 \\
\hline & & 9.15 & $\left.3310^{b}\right)$ & & & & & & \\
\hline & & 10.45 & $\left.2700^{b}\right)$ & 3350 & (3325) & $s_{1}+s_{2}$ & & & \\
\hline & $\frac{1}{2}^{-}$ & 0.0 & 3540 & & & & 3570 & $(3570)$ & 3560 \\
\hline & & 9.23 & $\left.3295^{b}\right)$ & & & & & & \\
\hline & & 11.29 & 3270 & 3560 & $(3560)$ & $s_{1}+s_{2}$ & & & \\
\hline \multirow[t]{6}{*}{17} & $\frac{5}{2}^{+}$ & 0.0 & 3540 & & & & 3495 & (3495) & 3500 \\
\hline & $\frac{1}{2}^{+}$ & 0.87 & 3165 & & & & 2995 & $(2940)$ & 2970 \\
\hline & & 5.73 & 3490 & & & & & & \\
\hline & & 6.36 & 3740 & 3130 & (2970) & $s_{1}+s_{3}$ & & & \\
\hline & $\frac{3}{2}^{+}$ & 5.08 & 3560 & & & & 3285 & $(3320)$ & 3300 \\
\hline & & 5.87 & 3490 & 3125 & (3155) & $s_{1}$ & & & \\
\hline \multirow[t]{2}{*}{27} & $\frac{5}{2}^{+}$ & 0.0 & 5590 & & & & 5610 & & 5610 \\
\hline & & 2.73 & 5505 & 5590 & & $s_{1}$ & & & \\
\hline \multirow[t]{2}{*}{29} & $\frac{1}{2}^{+}$ & 0.0 & 5725 & & & & 5705 & & 5700 \\
\hline & & 4.84 & 5650 & 5645 & & $s_{1}$ & & & \\
\hline \multirow[t]{2}{*}{31} & $\frac{1}{2}^{+}$ & 0.0 & 6225 & & & & 6250 & & 6250 \\
\hline & & 3.13 & 6170 & 6240 & & $s_{1}$ & & & \\
\hline \multirow[t]{2}{*}{33} & $\frac{3}{2}+$ & 0.0 & 6365 & & & & 6350 & & 6350 \\
\hline & & 2.31 & 6405 & 6350 & & $s_{1}$ & & & \\
\hline \multirow[t]{5}{*}{39} & $\frac{1}{2}^{+}$ & 2.50 & 7255 & & & & 7310 & (7310) & 7310 \\
\hline & & 4.10 & 7225 & 7315 & $\left.(7315)^{c}\right)$ & & & & \\
\hline & $\frac{8}{2}^{+}$ & 0.0 & 7305 & & & & 7315 & (7315) & 7430 \\
\hline & & 5.27 & 7170 & & & & & & \\
\hline & & 5.61 & 7185 & 7430 & $(7440)$ & $s_{1}+s_{2}$ & & & \\
\hline \multirow[t]{11}{*}{41} & $7^{-}$ & 0.0 & 7280 & & & & 7235 & (7230) & 7230 \\
\hline & & 2.96 & 7505 & & & & & & \\
\hline & & 3.53 & 7450 & 7230 & $(7220)$ & $s_{1}+s_{2}$ & & & \\
\hline & $\frac{3}{2}^{-}$ & 1.94 & 7050 & & & & 6710 & $(6640)$ & 6600 \\
\hline & & 2.46 & 7225 & & & & & & \\
\hline & & $\left.3.73^{d}\right)$ & 7325 & 6710 & $(6600)$ & $s_{1}+s_{3}$ & & & \\
\hline & & 4.60 & 7210 & 6725 & $(6605)$ & $s_{1}+s_{2}+s_{3}$ & & & \\
\hline & $\frac{1}{2}-$ & $3.94^{d, e}$ & 6805 & & & & & & 6390 \\
\hline & & $\left.3.61^{\mathrm{d}, \mathrm{c}}\right)$ & 7395 & & & & & & \\
\hline & & 4.75 & 7165 & 6400 & (6385) & $s_{1}+s_{3}$ & & & \\
\hline & $\frac{5}{2}-$ & 4.88 & 7345 & & & & & & \\
\hline
\end{tabular}

For notation see text. Here, $E_{\mathrm{ex}}{ }^{i}$ are in units of $\mathrm{MeV}$, and other quantities are in units of $\mathrm{keV} .^{\mathrm{a}}$ ) $A=$ 15, ref. ${ }^{27}$ ); $A=17$, ref. ${ }^{28}$ ); $A=27-41$, ref. ${ }^{29}$ ). ${ }^{\text {b) }}$ Ref. ${ }^{35}$ ). ${ }^{c}$ ) See subsect. 3.3.4. ${ }^{d}$ ) Ref. ${ }^{30}$ ). ${ }^{\text {e) }}$ Ref. ${ }^{31}$ ). 
number in parenthesis is the corresponding value generated by the Skyrme II interaction. The last column of the table shows the optimum value of the s.p. charge dependent energy chosen for each system (see the discussion in the subsequent sections).

3.3.1. The $A=15$ nuclei. Two different model wave functions are available for this system. Shukla and Brown ${ }^{33}$ ) include only the $2 \mathrm{p}-3 \mathrm{~h}$ state with isospins $\left(T_{\mathrm{p}}, T_{\mathrm{h}}, T\right)=\left(1, \frac{1}{2}, \frac{1}{2}\right)$. Their wave functions give the values $\varepsilon^{\text {theor }}\left(\frac{3}{2}^{-}\right)=3495 \pm 55$ $\mathrm{keV}$ and $\varepsilon^{\text {theor }}\left(\frac{1}{2}^{-}\right)=3605 \pm 20 \mathrm{keV}$. These values are obtained from eq. (3.4) using the $C_{s}^{i}$ from the model wave functions and $\Delta_{s}^{\mathrm{CE}}$ approximated by spin averages over the dominant shell-model components contained in the p- and h-parts of the model wave functions. The more detailed wave functions of Lie and Engeland ${ }^{34}$ ) include $2 \mathrm{p}-3 \mathrm{~h}\left(T_{\mathrm{p}}, \frac{1}{2}, \frac{1}{2}\right)$ states with both $T_{\mathrm{p}}=1$ and 0 as the major core excitations as well as a small component of the $4 \mathrm{p}-5 \mathrm{~h}$ state with $\left(0, \frac{1}{2}, \frac{1}{2}\right)$. The Lie-Engeland wave functions yield the values $\varepsilon^{\text {theor }}\left(\frac{3}{2}^{-}\right)=3465 \mathrm{keV}$ and $\varepsilon^{\text {theor }}\left(\frac{1}{2}{ }^{-}\right)=3570 \mathrm{keV}$.

The value for $\varepsilon^{\mathrm{av}}\left(\frac{1}{2}^{-}\right)$based on a three level model (with $i=1,2,3=1 \mathrm{p}, s_{1}, s_{2}$ ) agrees with $\varepsilon^{\text {theor }}\left(\frac{1^{-}}{2}\right)$ as calculated with the Lie-Engeland wave function. On the other hand, $\varepsilon^{\text {av }}\left(\frac{1}{2}{ }^{-}\right.$) based on a two level model (with $i=1,2=1 \mathrm{p}, s_{1}$, the appropriate choice for the Shukla-Brown wave function), using the levels at $E_{\text {ex }}^{i}=0.0$ and 9.23 $\mathrm{MeV}$, has the value $3670 \mathrm{keV}$ which does not agree with the value of $\varepsilon^{\text {theor }}\left(\frac{1^{-}}{2}\right)$ calculated with the Shukla-Brown wave function. We therefore conclude that the Lie-Engeland wave function gives the most accurate description of the core excitations and choose $3460 \mathrm{keV}$ as the optimum value for $\varepsilon\left(\frac{1}{2}^{-}\right)$. The observed $10 \mathrm{keV}$ difference between this optimum value and the Lie-Engeland value of $\varepsilon^{\text {theor }}\left(\frac{1}{2}^{-}\right)$may be explained in terms of the neglected cross terms in eq. (3.4).

The situation for the $\frac{3}{2}^{-}$state is not as good. The observed $659 \mathrm{keV}$ difference between $\mathscr{E}^{1}\left(\frac{3}{2}^{-}\right)$and $\mathscr{E}^{3}\left(\frac{3}{2}^{-}\right)$can not be explained by the core-excitation corrections. Also $\varepsilon^{\mathrm{av}}\left(\frac{3}{2}^{-}\right)$based on the three level model differs by $115 \mathrm{keV}$ from the Lie-Engeland value of $\varepsilon^{\text {theor }}\left(\frac{3}{2}^{-}\right)$. The values of $\varepsilon^{\text {av }}\left(\frac{3^{-}}{2^{-}}\right)$based on the two level model are $3625 \mathrm{keV}$ (DME) and $3600 \mathrm{keV}$ (SkII), which again differ by more than $100 \mathrm{keV}$ from the value of $\varepsilon^{\text {theor }}\left(\frac{3}{2}^{-}\right)$calculated with the Shukla-Brown wave function. We conclude that the assignments of the levels at $E_{\mathrm{ex}}^{i}$ of $9.15 \mathrm{MeV}$ and $10.45 \mathrm{MeV}$ [ref. ${ }^{35}$ )] as predominant $s_{1}$ and $s_{2}$ type core excitations may be open to question. However, since the LieEngeland wave function gives a good description for the core excitations in the $\frac{1}{2}^{-}$s.h. state, we believe that it also gives the best approximation for the core excitations in the $\frac{3}{2}^{-}$s.h. state and choose the Lie-Engeland value $\varepsilon^{\text {theor }}\left(\frac{3}{2}^{-}\right)$as the optimum value for $\varepsilon\left(\frac{3}{2}^{-}\right)$.

3.3.2. The $A=17$ nuclei. The $\frac{5}{2}^{+}, \frac{1}{2}^{+}$and $\frac{3}{2}^{+}$levels of this system have been studied by Brown and Green $\left.{ }^{13}\right)$. Without specifying the isospin $\left(T_{p}, T_{\mathrm{h}}, \frac{1}{2}\right)$ Brown and Green included much more complicated $3 \mathrm{p}-2 \mathrm{~h}$ and $5 \mathrm{p}-4 \mathrm{~h}$ states than those assumed in this work, while the correction seems to be insensitive to the details of the coreexcited states except for $T_{\mathrm{p}}, T_{\mathrm{h}}$. By assuming the $s_{1}$ and $s_{3}$ type core-excitations for the Brown-Green wave functions, we obtain the smallest values $\varepsilon^{\text {theor }}\left(\frac{5^{+}}{2}\right)=3455 \mathrm{keV}$, 
$\varepsilon^{\text {theor }}\left(\frac{1}{2}^{+}\right)=2995 \mathrm{keV}$ and $\varepsilon^{\text {theor }}\left(\frac{3}{2}^{+}\right)=3285 \mathrm{keV}$. On the other hand, assuming an equal amount $s_{1}$ and $s_{2}$ type core excitations for the $3 \mathrm{p}-2 \mathrm{~h}$ state and $s_{3}$ type core excitation for the $5 \mathrm{p}-4 \mathrm{~h}$ state, we obtain $\varepsilon^{\text {theor }}\left(\frac{5}{2}^{+}\right)=3500 \mathrm{keV}, \varepsilon^{\text {theor }}\left(\frac{1}{2}^{+}\right)=3050 \mathrm{keV}$ and $\varepsilon^{\text {theor }}\left(\frac{3}{2}^{+}\right)=3355 \mathrm{keV}$. Recently, Watt, Cole and Whitehead ${ }^{12}$ ) have studied the ${ }^{17} \mathrm{O}$ nucleus with a detailed shell-model calculation including all possible $2 \hbar \omega$ excitations of the ${ }^{16} \mathrm{O}$ core, with no truncation of the possible $(\mathrm{sd})^{3}(\mathrm{p})^{-2} 3 \mathrm{p}-2 \mathrm{~h}$ excitations. Although the details of their wave function are not published, they quote a $12.4 \%$ $2 \mathrm{p}-1 \mathrm{~h}$ admixture and a $9.4 \% 3 \mathrm{p}-2 \mathrm{~h}$ admixture in the ground state of ${ }^{17} \mathrm{O}$. The $2 \mathrm{p}-1 \mathrm{~h}$ admixture can be expected to be contained in our s.p. wave functions, since this is folded into our HF wave function. We therefore retain only the $9.4 \%$ admixture of the $3 \mathrm{p}-2 \mathrm{~h}$ core excitation in the $\frac{5}{2}^{+}$ground state. By assuming the $s_{1}$ type core excitation for the Watt-Cole-Whitehead wave function, we obtain the value $\varepsilon^{\text {theor }}\left(\frac{5}{2}^{+}\right)=3495$ $\mathrm{keV}$. By assuming an equal amount of $s_{1}$ and $s_{2}$ type core excitations for the $3 \mathrm{p}-2 \mathrm{~h}$ state, we obtain $\varepsilon^{\text {theor }}\left(\frac{s}{2}^{+}\right)=3520 \mathrm{keV}$.

Unfortunately, the experimental level assignment of this system is too poor to determine an averaged value $\varepsilon^{\mathrm{av}}(\alpha)$. The optimum value of $\varepsilon(\alpha)$ is obtained by averaging the smallest values $\varepsilon^{\text {theor }}(\alpha)$ obtained with the DME and the Skyrme II interaction, where we choose the $\varepsilon^{\text {theor }}\left(\frac{5^{+}}{2}\right)$ obtained by the Watt-Cole-Whitehead wave function and the $\varepsilon^{\text {theor }}\left(\frac{1}{2}^{+}\right)$and $\varepsilon^{\text {theor }}\left(\frac{3}{2}^{+}\right)$by the Brown-Green wave functions.

3.3.3. The $A=27,29,31$ and 33 nuclei. The core-excitation corrections are very small in these nuclear systems, so that the s.p. charge dependent energies $\varepsilon$ are not very sensitive to the details of the core excitations. It is mainly this fact which makes it possible to treat these systems as s.p. (s.h.) systems as far as their Coulomb energies are concerned ${ }^{36,37}$ ).

Theoretical values of the ${ }^{28} \mathrm{Si}$ core system are obtained from the shell-model calculations of Wildenthal and McGrory ${ }^{38}$ ). There are two different shell-model calculations for the ${ }^{32} \mathrm{~S}$ core system. The first one, given by Glaudemans et al. ${ }^{39}$ ), includes only $2 \mathrm{~s}_{\frac{1}{2}}-1 \mathrm{~d}_{\frac{3}{2}}$ configurations. The second, given by Wildenthal et al. ${ }^{40}$ ), includes $2 s_{\frac{1}{2}}-1 d_{\frac{3}{2}}$ configurations with excitations up to $1 d_{\frac{3}{2}}^{-2}$ from the $d_{\frac{3}{2}}$ space. However, both wave functions give almost identical values for the $\varepsilon(\alpha)$ to within $\pm 10 \mathrm{keV}$. Therefore we employ the averaged value obtained from the two models as the $\varepsilon^{\text {theor }}(\alpha)$. We choose the values of $\varepsilon^{\text {theor }}(\alpha)$ as the optimum values for $\varepsilon(\alpha)$.

3.3.4. The $A=39$ nuclei. Wiktor ${ }^{41}$ ) has studied the $A=39$ system using the intermediate coupling model. In his assignments the second $\frac{1}{2}^{+}$and $\frac{3}{2}^{+}$states are composed of a $1 d_{\frac{3}{2}}$ hole coupled to the $2^{+}$core excitation of the ${ }^{40} \mathrm{Ca}$ nucleus. Gerace and Green have shown that the lowest $2^{+}$state of ${ }^{40} \mathrm{Ca}$ is predominantly a $4 \mathrm{p}-4 \mathrm{~h}$ state $(87.4 \%)$. Therefore, the theoretical values, $\varepsilon^{\text {theor }}(\alpha)$, have been obtained by the combination of the two model wave functions given by Wiktor and by Gerace and Green $\left.{ }^{14}\right)$. The results are $\varepsilon^{\text {theor }}\left(\frac{1}{2}^{+}\right)=7310 \mathrm{keV}$ and $\varepsilon^{\text {theor }}\left(\frac{3}{2}^{+}\right)=7315 \mathrm{keV}$. Furthermore, if we employ these combined wave functions to determine the $\varepsilon^{\mathrm{av}}(\alpha)$, we obtain the values $\varepsilon^{\mathrm{av}}\left(\frac{1}{2}^{+}\right)=7315 \mathrm{keV}$ and $\varepsilon^{\mathrm{av}}\left(\frac{3}{2}^{+}\right)=7290 \mathrm{keV}$.

Since $\varepsilon^{\mathrm{av}}\left(\frac{1}{2}+\right)$ is in excellent agreement with $\varepsilon^{\text {theor }}\left(\frac{1}{2}{ }^{+}\right)$, we choose it as the optimum 
value for $\varepsilon\left(\frac{1}{2}^{+}\right)$. For the $\varepsilon\left(\frac{3}{2}^{+}\right)$, the combined Wiktor-Gerace and Green model may be in trouble because there is a third $\frac{3}{2}^{+}$level very closely and also because the correction $\left(\mathscr{E}^{11}\left(\frac{3}{2}^{+}\right)-\varepsilon^{\mathrm{av}}\left(\frac{3}{2}^{+}\right)\right)$cannot be explained with the core excitations. Consequently, we prefer the $\varepsilon^{a v}\left(\frac{3}{2}+\right)$ calculated with the three level model as the optimum value of $\varepsilon\left(\frac{3}{2}+\right)$ because it includes the Wiktor-Gerace and Green core excitation and also the nearby third level.

3.3.5. The $A=41$ nuclei. The $\frac{7}{2}^{-}$and $\frac{3}{2}^{-}$levels of this system have been studied by Gerace and Green ${ }^{14}$ ). They obtained two model wave functions. The first one includes the $3 \mathrm{p}-2 \mathrm{~h}$ state with $\left(T_{\mathrm{p}}, T_{\mathrm{h}}, T\right)=\left(\frac{1}{2}, 1, \frac{1}{2}\right)$, and it gives $\varepsilon^{\text {theor }}\left(\frac{1}{2}-\right)=7190 \mathrm{keV}$ and $\varepsilon^{\text {theor }}\left(\frac{3^{-}}{2}\right)=6885 \mathrm{keV}$. These values do not agree with the values of the $\varepsilon^{\text {av }}$ based on a two level model $(N=1)$. The second model wave function includes the $3 \mathrm{p}-2 \mathrm{~h}$ state with $\left(\frac{1}{2}, 1, \frac{1}{2}\right)$ and the $5 \mathrm{p}-4 \mathrm{~h}$ state with $\left(\frac{1}{2}, 0, \frac{1}{2}\right)$, and it gives $\varepsilon^{\text {theor }}\left(\frac{7}{2}^{-}\right)=7235 \mathrm{keV}$ and $\varepsilon^{\text {theor }}\left(\frac{3}{2}^{-}\right)=6710 \mathrm{keV}$. If we identify the third $\frac{7}{2}^{-}$level of ${ }^{41} \mathrm{Ca}$ as the level at $3.53 \mathrm{MeV}$ and that of ${ }^{41} \mathrm{Sc}$ at $3.70 \mathrm{MeV}, \varepsilon^{\mathrm{av}}\left(\frac{7}{2}{ }^{-}\right)$based on the three level model $(N=2)$ agree with $\varepsilon^{\text {theor }}\left(\frac{7^{-}}{2}\right)$. The value of $\varepsilon^{\text {theor }}\left(\frac{3}{2}^{-}\right)$agrees almost exactly with that of $\varepsilon^{\text {av }}\left(\frac{3}{2}^{-}\right)$based on a three level model $(N=2)$ using the DME. In addition to this fact, the value of $\varepsilon^{\mathrm{av}}\left(\frac{3}{2}^{-}\right)$based on a four level model $(N=3)$ is found to be very close to that of $\varepsilon^{\mathrm{av}}\left(\frac{3}{2}^{-}\right)$ based on a three level model. Although there is no theoretical work on $\frac{1}{2}^{-}$levels in this system, we find that the values of $\varepsilon^{\mathrm{av}}\left(\frac{1}{2}^{-}\right)$obtained are quite insensitive to the choice of $N$. Therefore, the value of $6390 \mathrm{keV}$, which is the average value of the $\varepsilon^{\text {av }}\left(\frac{1^{-}}{2}\right)$ obtained with the DME and the Skyrme II interaction, is chosen as the optimum value of the $\varepsilon\left(\frac{1}{2}^{-}\right)$.

Although the calculations with the Skyrme II interaction give results very similar to those with the use of the DME for the $\frac{7}{2}^{-}$and $\frac{1}{2}^{-}$states, there is a significant difference between the two interactions for the $\frac{3}{2}^{-}$states. We give preference to the values obtained with the Skyrme II interaction since only these values of $\varepsilon\left(\frac{3}{2}^{-}\right)$satisfy the relationship (3.12) with $N=2$ for the $\frac{7}{2}^{-}, \frac{3}{2}^{-}$and $\frac{1}{2}^{-}$levels. Unfortunately, we can not obtain any information for $\varepsilon\left(\frac{5}{2}^{-}\right)$. In addition to the lack of data of the experimental Coulomb displacement energies, the s.p. strength [from $(d, p)$ reactions etc.] is known to be badly fragmented over many levels. Moreover, the value of 7345 $\mathrm{keV}$ may not correspond to the Coulomb displacement energy of the state $E_{\mathrm{ex}}^{1}\left(\frac{5}{2}^{-}\right)$ [compare the similar situation for the case of $E_{\mathrm{ex}}^{1}\left(\frac{1}{2}^{-}\right)$, refs. $\left.{ }^{30,31}\right)$ ].

3.3.6. Summary. In general, the value of $\varepsilon^{\mathrm{av}}$ based on a three level model $(N=2)$ agrees almost exactly with the $\varepsilon^{\text {theor }}$ calculated with the model wave function based on a co-existence of the s.p. state and two core-excited states. The net core-excitation correction, which is defined by $\varepsilon^{\text {opt }}(\alpha)-\mathscr{E}^{\mathscr{1}}(\alpha)$, is always negative for all s.p. systems, while it is always positive for all s.h. systems. Generally, the core-excitation correction in the s.p. system has a different sign from that in the s.h. system with the same core. Although the core-excitation corrections are consequently important in obtaining a consistent description for both s.p. and s.h. systems, the core excitations cannot be responsible for removing the Nolen-Schiffer anomaly, $\varepsilon_{\text {exp }}^{\text {s.p. }}-\varepsilon_{\mathrm{calc}}$, since the anomaly, averaged over the s.p. and s.h. states in nuclei with the same core, is not changed 
much by the effect of the many-particle many-hole core excitation. For example, the anomalies without the core-excitation corrections (from table 2) are respectively 340 and $360 \mathrm{keV}$ for the $1 \mathrm{~d}_{\frac{3}{2}}$ and $1 \mathrm{p}_{\frac{1}{2}}^{-1}$ states of the ${ }^{16} \mathrm{O}$ core system. These give an averaged anomaly of $350 \mathrm{keV}$. On the other hand, these anomalies become respectively 300 and $380 \mathrm{keV}$ with the core-excitation corrections. Consequently, the averaged anomaly becomes $340 \mathrm{keV}$. Thus, the averaged anomaly changes only $10 \mathrm{keV}$ with the coreexcitation corrections, while the difference between the anomalies of both states changes from 20 to $80 \mathrm{keV}$.

TABLE 6

Anomalies of the s.p. Coulomb displacement energies in units of $\mathrm{keV}$

\begin{tabular}{|c|c|c|c|c|c|c|}
\hline \multirow[t]{2}{*}{$A$} & \multirow[t]{2}{*}{ State } & \multirow{2}{*}{$\varepsilon_{\text {exp }}^{\text {s.p. a }}$} & \multicolumn{2}{|c|}{ DME } & \multicolumn{2}{|c|}{ SkII } \\
\hline & & & $\varepsilon_{\text {tot }}^{\mathrm{eal}}$ & diff. & $\varepsilon_{\text {tot }}^{\mathrm{cal}}$ & diff. \\
\hline \multirow[t]{2}{*}{15} & $\frac{3}{2}^{-}$ & 3460 & 3215 & 250 & 3270 & 190 \\
\hline & $\frac{1}{2}-$ & 3560 & 3180 & 380 & 3270 & 290 \\
\hline \multirow[t]{3}{*}{17} & $\frac{8}{2}+$ & 3500 & 3200 & 300 & 3305 & 190 \\
\hline & $\frac{1}{2}+$ & $\left.2970(3130)^{b}\right)$ & 2905 & $\left.60(220)^{b}\right)$ & 2760 & 210 \\
\hline & $\frac{3}{2}{ }^{+}$ & 3300 & 2875 & 430 & 3030 & 270 \\
\hline 27 & $\frac{5}{2}+$ & 5610 & 5130 & 480 & 5115 & 490 \\
\hline 29 & $\frac{1}{2}+$ & 5700 & 5415 & 290 & 5465 & 240 \\
\hline 31 & $\frac{1}{2}+$ & 6250 & 5710 & 540 & 5685 & 560 \\
\hline 33 & $\frac{3}{2}+$ & 6350 & 5990 & 360 & 6070 & 280 \\
\hline \multirow[t]{2}{*}{39} & $\frac{1}{2}+$ & 7310 & 6940 & 370 & 7040 & 270 \\
\hline & $\frac{3}{2}{ }^{+}$ & 7430 & 6895 & 540 & 7000 & 430 \\
\hline \multirow[t]{4}{*}{41} & $\frac{7}{2}-$ & 7230 & 6790 & 440 & 6875 & 350 \\
\hline & $\frac{3}{2}-$ & $\left.6600(6710)^{b}\right)$ & 6330 & $\left.270(380)^{b}\right)$ & 6260 & 340 \\
\hline & $\frac{1}{2}-$ & 6390 & 5980 & 410 & 6055 & 330 \\
\hline & $\frac{5}{2}-$ & & 6355 & & 6645 & \\
\hline
\end{tabular}

2) The optimum value of the experimental s.p. charge dependent energy extracted from the experimental Coulomb displacement energies (see sect. 3).

b) The experimental s.p. charge dependent energy obtained with the DME.

The optimum values of the s.p. charge dependent energies are summarized in table 6 and compared with the s.p. energies calculated in sect. 2. The numbers in parentheses are included where the optimum value depends strongly upon the interaction (DME or SkII) used to calculate the core-excitation corrections. The anomalies obtained in table 6 are always positive and less state dependent in each mass system than those obtained in table 2 . Moreover, in table 6 , the remaining anomaly in the ground state of the s.p. system is always smaller than that in the ground state of the s.h. system with the same core. These relationships obtained in table 6 are the same as those obtained with the s.p. energies due to a short range force. This suggests that the remaining anomaly may be resolved with the introduction of some short range charge symmetry breaking force. 


\section{Discussion and conclusion}

\subsection{CHARGE SYMMETRY BREAKING FORCE}

Although many authors $6,8,42$ ) have attempted to explain the anomaly with the introduction of a CSB force, they have failed to explain the anomaly in a consistent manner. For instance, Shlomo ${ }^{8}$ ) showed that a CSB force, which is adjusted to fit the anomaly of ${ }^{3} \mathrm{He}-{ }^{3} \mathrm{H}$, is too weak to account for the anomaly of ${ }^{41} \mathrm{Sc}-{ }^{41} \mathrm{Ca}$, while it is too strong to explain the anomaly of ${ }^{15} \mathrm{O}-{ }^{15} \mathrm{~N}$. Such inconsistencies may stem from the fact that the calculated s.p. charge dependent energies have been compared with the raw experimental Coulomb displacement energies. With the analysis of sect. 3 the experimental s.p. charge dependent energies are now available to be compared with the calculated s.p. charge dependent energies. In this subsection we examine a phenomenological CSB force as a possible origin of the observed remaining anomaly. We define the phenomenological CSB force having a Yukawa radial shape in such a way that

$$
\begin{aligned}
& V^{\operatorname{CSB}}(1,2)=\left[\frac{1}{12}\left(3+\tau_{1} \cdot \tau_{2}\right)-\frac{1}{4}\left[\tau_{1}+\tau_{2}\right]_{0}^{1}+\frac{1}{2} \sqrt{\frac{1}{6}}\left[\tau_{1} \times \tau_{2}\right]_{0}^{2}\right] \\
& \times \sum_{n}\left[a_{n} f\left(\mu_{n}\right)+b_{n} g\left(\mu_{n}\right)\right],
\end{aligned}
$$

where

$$
f\left(\mu_{n}\right)=-\frac{v_{n}}{100} \frac{\mathrm{e}^{-\mu_{n} r_{12}}}{\mu_{n} r_{12}}, \quad g\left(\mu_{n}\right)=-\frac{v_{n}\left(\sigma_{1} \cdot \sigma_{2}\right)}{100} \frac{\mathrm{e}^{-\mu_{n} r_{12}}}{\mu_{n} r_{12}},
$$

with $\mu_{n}=n m_{n} c / \hbar$. Here the coefficients $a_{n}$ and $b_{n}$ are fitting parameters and $1 / \mu_{n}$ is a phenomenological exchange range corresponding to a particle with $n$ times the pion mass $\left(n m_{\pi}\right)$. To put potentials with different $n$ on a comparable basis, the $v_{n}$ is constrained by the condition

With the restriction ${ }^{43}$ )

$$
v_{n} / \mu_{n}^{3}=135 \mathrm{MeV} \cdot \mathrm{fm}^{3}
$$

$$
-\sum a_{\mathrm{n}}+3 \sum b_{\mathrm{n}}=0.0 \pm 0.8
$$

the interaction is consistent with the free $N-N{ }^{1} S_{0}$ scattering data. The s.p. energy due to the CSB force is given by

$$
\varepsilon^{\mathrm{CSB}}(\alpha)=\sum_{q}\left\langle\alpha q\left|V^{\mathrm{CSB}}\right| \alpha q\right\rangle_{\mathrm{a} . \mathrm{s.}} .
$$

Fig. 1 shows the $\varepsilon$-values calculated with the DME as a function of the range parameter $n$ for pure $f(\mu)$ and $g(\mu)$ type interactions. Values for both the ${ }^{16} \mathrm{O}$ and ${ }^{40} \mathrm{Ca}$ core systems are shown. The $\varepsilon$-values for the pure $f(\mu)$ type interaction are almost independent of the range parameter $n$. Also, the ratio of one $\varepsilon(\alpha)$ to another $\varepsilon(\beta)$ is insensitive to the choice of the range parameter $n$ for the pure $g(\mu)$ type interaction. Therefore, if the anomaly is resolved by a CSB force having $a_{l} f\left(\mu_{l}\right)$ and $b_{m} g\left(\mu_{m}\right)$ terms, it can also be resolved by another CSB force having $a_{l} f\left(\mu_{L}\right)$ and $b_{M} g\left(\mu_{M}\right)$ terms, where $b_{M}=b_{m}\left[\left\langle g\left(\mu_{m}\right)\right\rangle_{\alpha} /\left\langle g\left(\mu_{M}\right)\right\rangle_{\alpha}\right]$.The difference between these 


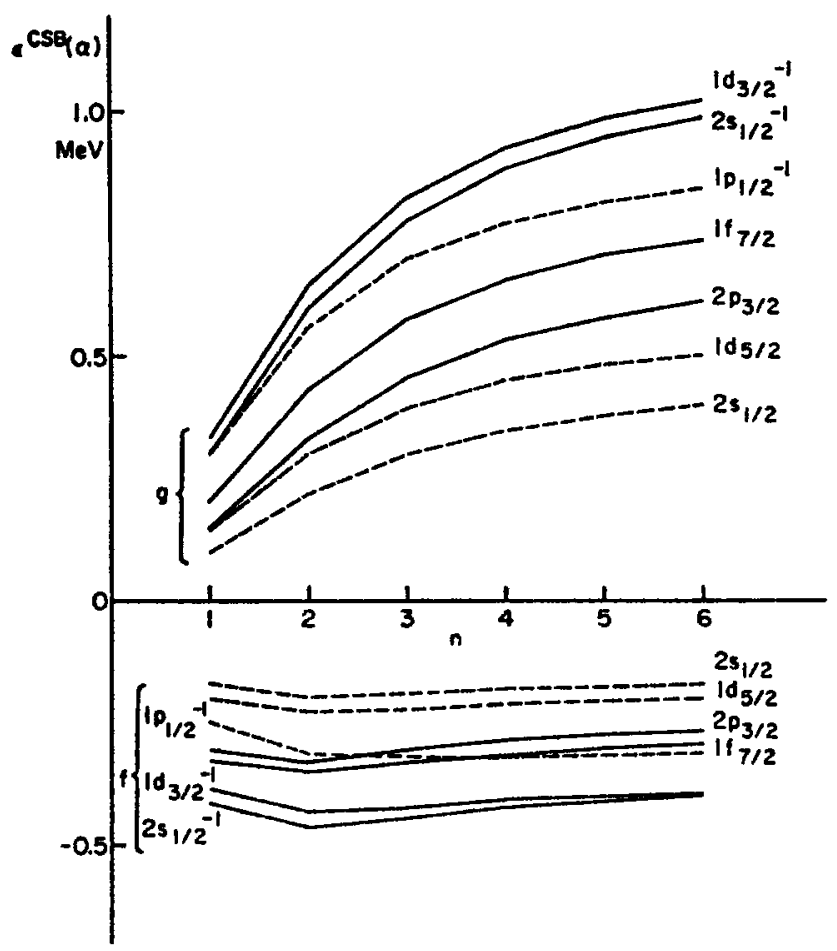

Fig. 1. The s.p. energies due to pure $f(\mu)$ and $g(\mu)$ type CBS forces calculated with the DME as a function of the range parameter $n$ in units of $\mu_{1}=m_{\pi} c / \hbar$. Energies of the $1 p_{\frac{1}{2}}^{-1}, 1 d_{\frac{s}{2}}$ and $2 s_{\frac{1}{1}}$ states of ${ }^{16} \mathrm{O}$ and the $2 \mathrm{~s}_{\frac{1}{2}}^{-1}, 1 \mathrm{~d}_{\frac{3}{2}}^{-1}, 1 \mathrm{f}_{\frac{3}{2}}$ and $2 \mathrm{p}_{\frac{3}{2}}$ states of ${ }^{40} \mathrm{Ca}$ are shown.

two CSB forces affects only the value of the left hand side of the restriction (4.3). These facts suggest that it is sufficient to examine a CSB force made from a combination of one $f\left(\mu_{l}\right)$ and one $g\left(\mu_{m}\right)$ type term. Assuming a CSB force of pure $f\left(\mu_{l}\right)$ type with a single term, we obtained $a_{1}=-1.33$ for $l=1$ and $a_{2}=-1.26$ for $l=2$ by adjusting the coefficient $a_{l}$ to fit the anomaly of the ground state of $A=41$ with the DME. For the anomalies of the ground states of the $A=15,17$ and 39 systems, the first CSB force gives the s.p. energies of 335,265 and $520 \mathrm{keV}$ respectively, while the second gives the values of 400,290 and $555 \mathrm{keV}$ respectively. The corresponding coefficients with the Skyrme II interaction are $a_{1}=-1.06$ and $a_{2}=-1.00$. For the anomalies of the ground states of the $A=15,17,39$ and 41 systems, the first CSB force gives the values of $275,220,415$ and $350 \mathrm{keV}$ respectively. The second gives the values of $340,240,440$ and $350 \mathrm{keV}$. For pure $f\left(\mu_{l}\right)$ type CSB force with $l \geqq 3$, the s.p. energies are almost the same as those with $l=2$. Table 7 shows the s.p. energies due to the CSB forces having one $f\left(\mu_{l}\right)$ and one $g\left(\mu_{m}\right)$ term, whose coefficients are adjusted to fit the anomalies of the ground state of $A=15$ and 41 . In general the anomalies of the ground states of mirror nuclei can be reproduced in a consistent manner with the introduction of the CSB force with the possible exception of the 
TABLE 7

The s.p. energies (keV) due to phenomenological CSB forces adjusted to fit the anomalies of the ground states of $A=15$ and 41

\begin{tabular}{|c|c|c|c|c|c|c|c|c|c|}
\hline \multirow[t]{2}{*}{$A$} & \multirow[t]{2}{*}{ State } & \multicolumn{4}{|c|}{ DME } & \multicolumn{4}{|c|}{ SkII } \\
\hline & & diff. & $\left.V_{1}{ }^{2}\right)$ & $\left.V_{2}^{b}\right)$ & $\left.V_{3}{ }^{c}\right)$ & diff. & $\left.V_{1}{ }^{9}\right)$ & $\left.V_{2}{ }^{e}\right)$ & $\left.V_{3}{ }^{\prime}\right)$ \\
\hline \multirow[t]{2}{*}{15} & $1 p_{\frac{3}{2}}-1$ & 250 & 395 & 405 & 405 & 190 & 300 & 300 & 295 \\
\hline & $1 p_{\frac{1}{2}}-1$ & 380 & 380 & 380 & 380 & 290 & 290 & 290 & 290 \\
\hline \multirow[t]{3}{*}{17} & $1 d_{\frac{s}{2}}$ & 300 & 275 & 285 & 285 & 190 & 225 & 235 & 235 \\
\hline & $2 s_{\frac{1}{2}}$ & $60(220)$ & 225 & 255 & 255 & 210 & 155 & 180 & 175 \\
\hline & $1 d_{\frac{3}{2}}$ & 430 & 150 & 150 & 150 & 270 & 135 & 130 & 130 \\
\hline 27 & $1 d_{\frac{5}{2}}^{-1}$ & 480 & 460 & 470 & 470 & & & & \\
\hline 29 & $2 s_{\frac{1}{2}}$ & 290 & 465 & 510 & 510 & & & & \\
\hline 31 & $2 s_{\frac{1}{2}}-1$ & 540 & 495 & 525 & 525 & & & & \\
\hline 33 & $1 d_{\frac{3}{2}}$ & 360 & 490 & 480 & 480 & & & & \\
\hline \multirow[t]{2}{*}{39} & $2 s_{\frac{1}{2}}-1$ & 370 & 575 & 585 & 590 & 270 & 440 & 450 & 455 \\
\hline & $1 d_{\frac{3}{2}}-1$ & 540 & 550 & 540 & 540 & 430 & 420 & 405 & 410 \\
\hline \multirow[t]{4}{*}{41} & $1 f_{\frac{7}{2}}$ & 440 & 440 & 440 & 440 & 350 & 350 & 350 & 350 \\
\hline & $2 \mathrm{p}_{\frac{3}{2}}$ & $270(380)$ & 400 & 420 & 425 & 340 & 295 & 320 & 320 \\
\hline & $2 p_{\frac{1}{2}}$ & 410 & 340 & 355 & 360 & 330 & 250 & 255 & 255 \\
\hline & $1 \mathrm{f}_{\frac{6}{2}}$ & & 345 & 340 & 340 & & 295 & 290 & 290 \\
\hline
\end{tabular}

Diffs. are taken from table 6.

$V_{1}=a_{1} f\left(\mu_{1}\right)+b_{1} g\left(\mu_{1}\right), \quad V_{2}=a_{2} f\left(\mu_{2}\right)+b_{1} g\left(\mu_{1}\right), \quad V_{3}=a_{2} f\left(\mu_{2}\right)+b_{2} g\left(\mu_{2}\right)$,

where $f\left(\mu_{n}\right)=-\left(v_{n} / 100\right)\left(\exp \left(-\mu_{n} r / \mu_{n} r\right)\right.$ and $g\left(\mu_{n}\right)=\left(\sigma_{1} \cdot \sigma_{2}\right) f\left(\mu_{n}\right)$ with $v_{n}=135 \mu_{n}^{3} \mathrm{MeV}$ and $\mu_{n}=$ $n m_{\pi} c / \hbar$.

a) $\left(a_{1}, b_{1}\right)=(-1.14,0.31)$ b) $\left(a_{2}, b_{1}\right)=(-1.37,-0.19)$. $\quad\left(a_{2}, b_{1}\right)=(-1.42,-0.13)$

d) $\left(a_{1}, b_{1}\right)=(-1.00,0.09)$. c) $\left(a_{2}, b_{1}\right)=(-1.26,-0.43)$. $\quad$ f $\left(a_{2}, b_{2}\right)=(-1.36,-0.29)$.

mass 29 and 33 systems. The CSB force which resolves the anomaly in the ground states also gives a good fit for the s.p. states of higher excitation energy, particularly for the $2 \mathrm{p}_{\frac{1}{2}}$ and $2 \mathrm{p}_{\frac{1}{2}}$ states of $A=41$. The predictions for the $1 \mathrm{~d}_{\frac{2}{2}}$ state in $A=17$ are very sensitive to the exact nature of the HF wave function and core-excitation corrections which are uncertain due to poor level assignments. However, it is almost impossible to reproduce the anomalies of the deeper hole states with the same CSB force. The amount of the CSB force is about $1 \%$ of the charge independent nuclear force, and is not inconsistent with the present free $\mathrm{N}-\mathrm{N}^{1} \mathrm{~S}_{0}$ scattering data. Although we have examined a spin-orbit type contribution to the CSB force, we find that it does not play an important role in the remaining anomalies of the ground states of mirror nuclei.

\subsection{CONCLUSION}

Although the use of the best available $\mathrm{HF}$ wave functions and the introduction of the core-excitation corrections have made the observed Nolen-Schiffer anomalies in the $T=\frac{1}{2}$ s.p. and s.h. systems more systematic and less state dependent, the overall 
magnitude of the observed anomalies cannot be reduced with these refinements. We therefore conclude that a CSB force or another effect is needed to resolve the anomaly. A simple phenomenological CSB nucleon-nucleon force, which is not inconsistent with the present free $\mathrm{N}-\mathrm{N}{ }^{1} \mathrm{~S}_{0}$ scattering data, can account for the observed anomalies of the ground states of s.p. and s.h. systems. This force also accounts for the observed anomalies in the higher excited s.p. states, while those of the deeper s.h. states need further explanation.

The author would like to thank Prof. K. T. Hecht for stimulating discussions and critical reading of the manuscript. He also would like to thank Prof. J. W. Negele, who suggested this problem and made the HF code accessible. He is grateful to Profs. K. Yazaki, J. Jänecke, N. Sherman and Dr. S. Shlomo for their helpful discussions.

\section{References}

1) J. Jänecke, Isospın in nuclear physics, ed. D. H. Wılkınson (North-Holland, Amsterdam, 1969) p.299

2) K. Okamoto, Phys. Lett. 11 (1964) 150

3) J. A. Nolen, Jr. and J. P. Schiffer, Phys. Lett. 29B (1969) 396

4) J. A. Nolen Jr. and J. P. Schiffer, Ann. Rev. Nucl. Sci. 19 (1969) 471

5) E. H. Auerbach, S. Kahana and J. Weneser, Phys. Rev. Lett. 23 (1969) 1253

6) J. W. Negele, Nucl. Phys. A165 (1971) 305

7) N. Auerbach, J. Hüfner, C. M. Shakin and A. K. Kerman, Rev. Mod. Phys. 44 (1972) 48

8) S. Shlomo, Phys. Lett. 42B (1972) 146

9) G. E. Brown, V. Horsfjord and K. F. Liu, Nucl. Phys. A205 (1973) 73

10) J. W. Negele, Int. Conf. on nuclear structure and spectroscopy, Amsterdam, 1974 (MIT, Center for theoretical physics publication, no. 431)

11) N. Lo Iudice, D. J. Rowe and S. S. M. Wong, Nucl. Phys. A219 (1974) 171

12) A. Watt, B. J. Cole and R. R. Whitehead, to be published

13) G. E. Brown and A. M. Green, Nucl. Phys. 75 (1966) 401

14) W. J. Gerace and A. M. Green, Nucl. Phys. A93 (1967) 110

15) J. W. Negele and D. Vautherin, Phys. Rev. C5 (1972) 1472

16) D. Vautherın and D. M. Brink, Phys. Rev. C5 (1972) 626

17) J. W. Negele and D. Vautherin, unpublished

18) N. V. Giai, D. Vautherin, M. Veneroni and D. M. Brınk, Phys. Lett. 35B (1971) 135

19) M. Abramowitz and I. A. Stegun, ed., Handbook of mathematical functions (National Bureau of Standard, Washington, DC)

20) B. Buck and P. E. Hodgson, Phil. Mag. 6 (1961) 1371

21) W. J. Romo, Nucl. Phys. A116 (1968) 618

22) H. Horie and K. Sasaki, Prog. Theor. Phys. 25 (1961) 475

23) A. de-Shalit and I. Talmi, Nuclear shell theory (Academic Press, New York, 1963)

24) S. Shlomo, Ph. D. thesis, Weizmann Institute (1973) $73 / 21 \mathrm{Ph}$

25) G. F. Bertsch and S. Shlomo, Phys. Rev. C10 (1974) 931:

S. Shlomo, private communication

26) A. Arima and S. Yoshida, Nucl. Phys. A161 (1971) 492

27) F. Ajzenberg-Selove, Nucl. Phys. A152 (1970) 1

28) F. Ajzenberg-Selove, Nucl. Phys. A166 (1971) 1

29) P. M. Endt and C. van der Leun, Nucl. Phys. A214 (1973) 1

30) W. M. Wilson, Jr. et al., Nucl. Phys. A227 (1974) 277

31) D. C. Kocher and W. Haeberli, Nucl. Phys. A196 (1972) 225

32) P. J. Ellis and E. Osnes, Phys. Lett. 41B (1972) 97 
33) A. P. Shukla and G. E. Brown, Nucl. Phys. A112 (1968) 296

34) S. Lie and T. Engeland. Nucl. Phys. A169 (1971) 617

35) C. E. Steerman and F. C. Young, Nucl. Phys. A192 (1972) 353

36) R. Sherr and I. Talmi, Phys. Lett. 56B (1975) 212

37) R. Sherr, to be published

38) B. H. Wildenthal and J. B. McGrory, Phys. Rev. C7 (1973) 714

39) P. W. M. Glaudemans, G. Wiechers and P. J. Brussard, Nucl. Phys. 56 (1964) 548

40) B. H. Wildenthal, J. B. McGrory, E. C. Halbert and H. D. Graber, Phys. Rev. C4 (1971) 1708

41) S. Wiktor, Phys. Lett. 40B (1972) 181

42) S. Shlomo and D. O. Riska, to be published

43) E. M. Henley, in Isospin in nuclear physics, ed. D. H. Wilkinson (North-Holland, Amsterdam, 1969) p. 17 